Cinémas

Revue d'études cinématographiques

Journal of Film Studies

\title{
Langage cinématographique et faillibilité
}

\section{Lucie Roy}

Volume 4, numéro 3, printemps 1994

Questions sur l'éthique au cinéma

URI : https://id.erudit.org/iderudit/1001040ar

DOI : https://doi.org/10.7202/1001040ar

Aller au sommaire du numéro

\section{Éditeur(s)}

Cinémas

\section{ISSN}

1181-6945 (imprimé)

1705-6500 (numérique)

Découvrir la revue

Citer cet article

Roy, L. (1994). Langage cinématographique et faillibilité. Cinémas, 4(3), 99-118. https://doi.org/10.7202/1001040ar
Résumé de l'article

Empruntant la voie d'une étude phénoménologique du langage cinématographique, l'auteure se penche sur la question de relative inhérence de la pensée en la tenant, cette inhérence, comme l'expression d'une sorte de Dasein ou d'un être-là du discours filmique. Décrivant, en quelque sorte, le site ou l'état de la pensée dans le discours, l'auteure aborde la problématique de la référentialité ou celle encore, en bout de course, des genres du discours filmique qui, sous le couvert d'une sorte de mondanéité, fait défiler le monde et la pensée. Bref, l'auteure considère que, même lorsqu'il joue du simulacre de leur dispersion ou de leur disparition, le cinéma participe de l'expression de la pensée et des réalités. Il impose, ce faisant, un constant questionnement éthique. 


\section{Langage cinématographique et faillibilité ${ }^{1}$}

\section{Lucie Roy}

\section{RÉSUMÉ}

Empruntant la voie d'une étude phénoménologique du langage cinématographique, l'auteure se penche sur la question de relative inhérence de la pensée en la tenant, cette inhérence, comme l'expression d'une sorte de Dasein ou d'un être-là du discours filmique. Décrivant, en quelque sorte, le site ou l'état de la pensée dans le discours, l'auteure aborde la problématique de la référentialité ou celle encore, en bout de course, des genres du discours filmique qui, sous le couvert d'une sorte de mondanéité, fait défiler le monde - et la pensée. Bref, l'auteure considère que, même lorsqu'il joue du simulacre de leur dispersion ou de leur disparition, le cinéma participe de l'expression de la pensée et des réalités. Il impose, ce faisant, un constant questionnement éthique.

\section{ABSTRACT}

By means of a phenomenological study of cinematographic language, the author examines the question of the relative inherentness of thought, which she considers to be the expression of a sort of Dasein or "beingthere" in filmic discourse. Describing, in a way, the site or the state of thought in discourse, she explores the problematic of referentiality, ultimately that of the genres of filmic discourse, which, under cover of a sort of worldliness, brings both the world and thought under scrutiny. In short, the author believes that even when cinema resorts to the simulacrum of their dispersal or disappearance, it participates in the expression of thought and of realities. Doing so, it imposes a constant ethical questioning. 
Une "retombée " dans le non-sens, l'indicible et la violence demeure notre possible indépassable.

Maurice Merleau-Ponty ${ }^{2}$

Le champ d'enquête de l'éthique déborde, on l'a dit dans le texte de présentation, celui de la morale. Englobante, l'éthique interroge à la fois le langage en tant qu'intériorité, visée symbolique et, donc, extériorité de la et des pensées. Et elle se préoccupe, ou peut se préoccuper au versant d'une éthique communicationnelle ou d'une philosophie politique, de l'ordre moral qui vient, si l'on veut, par et à la suite du langage. On le voit, l'éthique en question ne réfere pas à une pure existentialité ${ }^{3}$ ou à un pur examen de la métaphysique qui comprendrait l'étude des régions de la pensée, mais elle examine la manifestation, l'état de la pensée dans le langage. L'éthique ou, étymologiquement, l'éthos tend ici à questionner l'usage et le séjour de la pensée dans le discours ${ }^{4}$ et elle tend aussi à considérer le pathos du discours, la faillibilité ${ }^{5} \mathrm{du}$ langage, en tant qu'il peut constituer une sorte de moule et d'arrêtés pour la pensée. Car, «nous le pressentons, l'arbitraire de la signification requiert, pour être signifiant, l'arbitraire des volontés consensuelles qui en arrêtent la dérive. Ainsi les repères langagiers sont-ils équivalemment des repères éthiques, émergeant d'une herméneutique immanente au dialogue" (Houziaux, p. $28^{6}$ ). L'éthique concerne, en somme et en ce sens, non seulement le langage, mais elle se soucie également du fait de générer un arbitraire de comportement par, précisément, et notamment un arbitraire de langage - on dit notamment dans la mesure où on peut effectivement penser que des comportements non langagiers en génèrent d'autres ayant un même caractère arbitraire?

Posant tout d'abord l'idée des moments langagiers du cinéma dans lequel l'on peut admettre l'existence, encore à définir, d'une pensée en travail dans la fiction, est tentativement posée, sous l'angle de la phénoménologie, l'hypothèse d'un être du discours filmique en le tenant, et ceci est particulier, comme l'expression d'une sorte de Dasein ${ }^{8}$ - ou, tel que défini par Heidegger, d'un être-là, celui-ci scriptural : lieu et lien mémoriels - 
ou, par idéal, "mémorial ${ }^{9}$ " d'une sorte d'étendue de monde, en quelque sorte dévoilé par l'apparence de mondanéité ${ }^{10}$ et, j'ajoute, de momentanéité que ménage le circuit filmique.

Avant d'ébaucher quelques définitions relatives aux termes ici en usage, certaines précisions s'imposent au regard de la perspective d'enquête empruntée. Il est habituel de regarder du dehors du texte pour décrire - l'approche est sémiotique — les formes employées par les textes, mais il est plus rare de tenter de décrire - l'approche est plus inspirée par la phénoménologie que par la sémiotique - ce qui est décrit par les formes du texte observé. Or, au cinéma, ce qui est (d)écrit, c'est cette sorte d'étendue de monde, de mondanéité, à laquelle on vient de faire référence et celle-ci - c'est là sa définition - ne correspondrait pas uniquement à la prise de l'espace et du temps par l'entreprise textuelle du film, mais à une saisie théorique qui, de l'ordre de la représentation des comportements, comprend celui d'écrire et enjoint la réalité et la pensée à transiter dans le film.

Il est donc ici question d'un Dasein qui n'est pas d'existentialité et dont le là de l'être-là ne vient pas expliciter l'être en ses divers modes d'être-à: au-monde, au-langage. Mais il s'agit bien plutôt de considérer provisoirement dans le langage ici cinématographique la pure expressivité, un étant manifesté, un être-là dévoilé par le langage.

La mondanéité correspondrait pour le cinéma à cet apparaître premier, le plus évident ou le plus manifeste, c'est-à-dire à la photographie visuelle et, en quelque sorte, sonore comprise dans le film et, par elle notamment, à l'empreinte du réel dans le film. Presque comme dans l'expérience commune, l'espace, ici et ainsi photographié, se trouve traversé par un regard, une pensée, un être-là. On le voit, on fait ici glisser les termes d'une entreprise phénoménologique, qui concerne la description des phénomènes et l'explication de l'être des phénomènes, vers la description des phénomènes décrits par le film au-devant desquels ou derrière lesquels se meut une pensée. L'être-là filmique n'est, d'évidence, pas celui de la quotidienneté et il n'est pas davantage celui de la subjectivité. Encore que celle-ci peut-être reconnaissable: les films de Steven Spielberg et de Woody Allen sont effectivement assez nettement autographiés. Dans la présente 
entreprise, le Dasein - on reprend ce terme de Heidegger sans suivre le parcours ontico-ontologique de son entreprise - fait simplement écho à une présence, à une identité dans l'écriture, à une identité en écriture. L'ayant posée, on se propose, au versant d'une étude éthique du langage cinématographique, de questionner cette identité de l'écriture ou, pour regagner l'habituel terrain de l'analyse, d'interroger le sens du et des textes qu'est, on le souligne, son produit.

Il s'agit en somme, et ce n'est pas peu dire, de s'autoriser à inverser l'usuelle problématique phénoménologique. Plutôt, en effet, que de s'interroger sur l'être, les régions de sa pensée et son idéation par la parole, il s'agit de considérer le langage cinématographique et, par lui, la pensée en trace dans le film. On le voit, cette approche tend a priori à soutenir, pour en justifier l'approche, une sorte d'idéalisme de l'expressivité filmique et, par lui, un certain idéalisme éthique à questionner. À la façon de Heidegger, qui s'est longuement interrogé sur la parole, on pourrait croire que "le langage est la maison de l'Être" (Heidegger, 1946, p. 74) et ainsi se permettre la remontée du langage vers l'être ou, mieux pour ce qui nous concerne, vers la pensée.

Pensant la trace de la pensée dans le film ou l'identité en écriture dans le film, on pourrait ajouter à l'opposition de cet idéalisme de l'expressivité filmique que le questionnement éthique nuance une autre opposition, qui tient au simulacre de l'énonciation filmique. On le sait suffisamment, le film tend à faire jouer le non-apparaître de l'énonciation et le simulacre d'une immanence fictionnelle. Ricœur aurait ajouté à cette étude celle de la symbolique et aurait ainsi pu lire dans le film la réinterprétation de la faillibilité, de la finitude et de l'aveu qui comprennent l'idée de la mort et, autrement, celle des limites de la connaissance éthique humaine, en tant qu'elles participent, de son avis, de la trame et de la Métaphore vive" des récits.

Or, précisément, on pourrait croire que cet état de simulacre fictionnel et, par lui, celui d'un non-apparaître énonciatif comme, dans une autre perspective et pour prolonger encore un peu la parenthèse précédente, l'apparition d'un noyau symbolique, filmique, qui fait plus souvent qu'autrement jouer la menace, la peur et la mort - justifient pleinement une sorte d'her- 
méneutique des textes filmiques et appellent, par conséquent, une réflexion éthique.

On ne saurait davantage opposer à un questionnement éthique, qui cible sa réflexion sur une pensée en travail dans le film, le fait que le produit filmique est le fruit du travail de plusieurs intervenants (réalisateur, scénariste, etc.) dans la mesure où il réfere à une écriture, à l'identité d'une écriture. Le film fournit, en effet, presque toujours la sensation d'une certaine cohésion narrative et, pour le spectateur, les raisons de la rétablir. Il ne faudrait pas croire, cela dit, que jouant le simulacre et la dissimulation, la pensée disparaît, réellement, et qu'elle se trouve, face à un éventuel questionnement éthique, innocentée. Il est, à cet égard, curieux de constater que, considérant la fiction comme irréelle, on en arrive à irréaliser sa feinte et son jeu énonciatif qui sont pourtant bien réels.

On pose en ce qui concerne le présent exercice - la perspective phénoménologique et langagière ici engagée l'autorise - l'a priori du travail de la pensée dans l'écriture filmique qui, comme on vient de le voir, étudie le phénomène de l'apparaître filmique - et non, la différence est marquante, l'apparence filmique. Et on considère le perpétuel jeu de balancier ou le principe d'inter-férence des couches référentielles du film. L'une d'elles est syntaxique et narrative et constitue l'univers du récit filmique - le réseau du système d'analyse pourrait être, dans le cadre d'un autre projet, sémiotique et narratologique. L'autre est phénoménale et est constitutive d'une certaine forme de mondanéité et de momentanéité par laquelle, on verra comment, s'opère le mouvement de provenance et d'advenir du sens de l'univers du récit filmique - le réseau d'analyse est ici inspiré par une phénoménologie du langage, mais il pourrait autrement être motivé par des perceptions sociologique, anthropologique et historique qui contribueraient à dévoiler le sens du récit.

Ayant ainsi reconnu le réseau de cette intrication référentielle et, davantage, la référence ici faite à l'émergence d'une pensée en travail dans le film, on souhaite tisser la trame d'un questionnement sur l'éthique au cinéma en s'inspirant, le plus souvent de loin, de certaines réflexions phénoménologiques de Heidegger pour sa définition du Dasein, de Wittgenstein pour son approche 
philosophique du langage, de Ricœur pour sa conception du récit historique et de fiction.

Trois voies d'analyse sont, en somme et en clair, ici proposées. La première concerne, comme on vient de le voir, l'examen d'un Dasein - mais scriptural, ou d'un être-là du discours filmique. La deuxième, enchâssée dans la première, examine les questions de mondanéité et de momentanéité ou d'apparaître de l'écriture filmique. La troisième, enfin, veut proposer l'ouverture à une interrogation qui repose sur la faillibilité du discours filmique, sur celle d'une pensée en travail dans le discours filmique.

On veut, en somme, vérifier l'assertion qui veut que, se trouvant plus souvent qu'autrement voilée, dissimulée ou narrativement simulée au cinéma, la pensée, comme d'une autre façon, la réalité qu'elle convoque, risque de retomber dans le non-sens, l'indicible et, en bout de course, la violence.

\section{Un Dasein, mais scriptural, ou un être-là du discours filmique}

Nous parlons sans cesse, même quand nous ne proférons aucune parole, et que nous ne faisons qu'écouter ou lire (Heidegger, 1984, p. 13).

On l'a dit, ma démarche ne suit pas, quant au terme de $D a$ sein emprunté à Heidegger, une perspective ontico-ontologique de l'être ou celle encore, pour la définir très sommairement, de la génétique du recouvrement par l'étant de la totalité de l'êtrelà, à travers monde (mondanéité), temps (temporellité), histoire (historialité) et, plus loin, langage ${ }^{12}$.

On souhaite cependant s'inspirer, pour le cinéma, du concept de mondanéité, en tant, c'est là son intérêt, qu'il est traversé par la pensée et que, c'est là sa définition, la mondanéité est, en tant que l'être-là est au monde. En forme d'un avoir-été-de-l'être-là, la pensée semble apparaître, pour le cinéma, dans l'avoir-été-là tel que défini, pour la photographie, par Barthes dans son ouvrage La Chambre claire ${ }^{13}$. Or, si la première manifestation correspond au Dasein, au regard déjà posé qui se manifeste inlassablement dans l'espace de la photographie, la seconde, qui inclut la première, l'avoir-été-là constitue cet espace, comprend ce lieu luimême qui, un jour, a été saisi là. 
En clair, on ne s'interdit pas de mettre en rapport une présence, abstraite, celle d'un avoir-été-de-l'être-là, cristallisée dans l'espace de la scène, avec l'avoir-été-là d'un lieu, concret, inscrit dans l'espace de la pose mouvante du film. Pour dire vrai, et pour définir ce terme d'être-là, c'est précisément la rencontre de cet état de voyance placé en creux de l'espace cinématographique qui marque ses indices. Bref, la mondanéité existe en tant qu'elle est considérée comme un espace de monde visité par le là de l'être-là. Comme s'il participait d'une écriture phénoménale ou de l'ordre de la quotidienneté, le monde cinématographique se transforme en mondanéité sitôt, et c'est toujours le cas, que le Dasein le traverse, même pour y jouer le simulacre de son oblitération.

"Si maintenant nous nous interrogeons sur les données phénoménales que la connaissance manifeste elle-même, nous avons à retenir que la connaissance se fonde d'emblée sur un être-déjàauprès-du-monde, qui est aussi un constitutif essentiel pour l'être de l'être-là " (Heidegger, 1965, p. 85). On le voit, on tient le cinéma pour un art phénoménal, en tant qu'il permet la rencontre des phénomènes, et pour un art langagier, en tant qu'il est l'expression du phénomène de la pensée par l'apparence de mondanéité que ménage le circuit filmique.

Cela dit encore, on aurait pu différencier davantage ce terme de mondanéité et ajouter à cette notion celle de la spatialité. Dans cette perspective, la spatialité trouverait son expression par l'iconicité mouvante du film, qui emporte des moments proxémiques correspondant, si l'on veut, à la distance d'un Dasein, en description dans [et par rapport à] l'espace de la scène filmée. Ces moments proxémiques ne correspondraient pas simplement à la distance physique mais, plus loin, à l'apparaître de cette distance, à son sentiment, au sens de la distance et de la proximité. Ici comme ailleurs: "Un chemin "objectivement" plus long peut être plus court qu'un chemin "objectivement" très court, si ce dernier est "un véritable calvaire" et s'il paraît à celui qui le parcourt infiniment long" (Heidegger, 1965, p. 135). Ce qui fait sens, ce n'est donc pas la capacité de mettre à distance ou à proximité un "ici” et un "là». Ce qui fait sens, c'est la pensée qui traverse cette spatialité, cet "ici » et ce "là ". À la mondanéité, 
manifestée par l'écranicité du film, correspondrait l'être-là dans le monde ou l'être-au-monde et, c'est là sa particularité, sa voyance ou son expression par le discours filmique. Ajoutant le préfixe pré à la voyance filmique, on aurait pu lire dans l'expression de la spatialité filmique la pré-voyance, le recouvrement par la pensée de la pré-voyance filmique. En sorte que, devançant la mise en espace du film, c'est la pensée qui le sémantise. Le discours filmique emporte, en somme, presque directement un matériau de monde et une pensée en travail dans ce même matériau.

Or, comme on ne saurait concevoir la présence de cet apparaître de la mondanéité sans penser sa temporalisation par l'écriture, il vient aussitôt à l'esprit le mouvement d'une momentanéité qui traverse l'espace filmique et est, à l'évidence, traversée par cet être-là - Heidegger aurait, dans une autre perspective, employé le terme de temporellité et aurait, ce faisant, fait référence non seulement à la présence de l'être dans le temps, mais au caractère essentiellement temporel de l'être-là (Heidegger, 1986, p. 43).

Afin d'établir une sorte de parallèle entre la temporellité et la temporalité filmique, l'analyse pourrait porter sur l'émergence des silences sonores et écraniques en tant qu'ils sont constitutifs d'une sorte de temporalisation de la pensée dans l'écriture filmique, en tant, donc, que la manifestation de la pensée par le discours filmique est essentiellement temporelle. On considérerait, par conséquent, les silences sonores et écraniques comme des marques d'une écriture en acte et on s'éloignerait de l'idéalisation du silence et de la déception langagière qui en découle. "L'ouïr et le silence sont des possibilités, disait Heidegger, de la parole du discours. Ces phénomènes permettent seuls une explication complète du rôle fonctionnel que remplit le discours pour l'existentialité de l'existence" (Heidegger, 1965, p. 199-200).

La pensée ainsi considérée, phénoménale ou en travail dans le site du discours filmique, participerait d'une spatialisation, d'une certaine mondanéité de photographie en tant qu'elle décrit le monde et les rapports de la pensée avec le monde - et par ailleurs avec l'écriture. Et elle participerait, cette pensée, d'une temporalisation, d'une certaine momentanéité syntaxique 
et narrative. Celle, une fois encore, du monde inscrit dans l'univers du film et celle, notamment, de la (pré)voyance qui en autorise l'émergence, celle, bref, d'un Dasein qui court et fait courir l'écriture dans le film.

Au regard du faisceau d'enquête ici proposé, que des réflexions d'ordre phénoménologique éclairent, il appert que déracinée du monde par la narrativité et la syntaxe, l'écriture du film de fiction advient, mais continue de provenir ${ }^{14}$ du monde et de la pensée. Il s'agit, en fait et pour être plus clair, de réfléchir sur la vieille notion d'immanence au cinéma et il s'agit de la penser en forme d'effeuillement ou de fractionnement. Voici que se dessinent des lieux sur la surface écranique et que s'énoncent des temps: une syntaxe. Voilà que par elle, le récit, comme tiré audevant de l'écran, advient. Voici que se dessinent des lieux sur la surface écranique et que s'énoncent des temps: une syntaxe. Voilà que par elle, le récit, comme tiré vers l'arrière rejoint le monde et la réalité d'où le monde écranique provient. Voilà que le film trace le mouvement de sa provenance. "L'espace [même filmique] ne peut se comprendre qu'à partir du monde" (Heidegger, 1965, p. 143).

Bifurquant de l'analytique des horizons de l'être telle que proposée par Heidegger, on n'a qu'à faire correspondre, de façon provisoire, ce paradoxe du lointain qui rapproche, pour comprendre que, sur les plans de la photographie et notamment de l'écriture de la fiction, le monde et la pensée se trouvent, au cinéma, près parce qu'ils sont loin. Dit autrement, en forme d'advenir du monde par la pensée, en forme donc de propulsion par la pensée de la fiction à l'écran, le film trace toujours le réseau de sa provenance, celui du monde et de la pensée qui, comme en écho, persiste à le traverser. "Le Dasein "est" [précisément] son passé de la manière où son être, pour le dire rudimentairement, "advient" chaque fois à partir de son avenir" (Heidegger, 1946, p. 45). Cela dit enfin, à l'instar de Heidegger, "[...] nous n'entendons ni quelque chose comme l'être-éloigné (proximité), ni même la distance. Nous employons l'expression d'éloignement dans une signification active et transitive" (p. 145). Et encore:

Lêtre-là est spatial par le fait que, dans la préoccupation prévoyante [ou, ici, pré-voyante ou préfigurante de 
la pensée dans l'espace du discours filmique], il découvre l'espace, en telle sorte qu'il se rapporte constamment aux étants en les éloignant; c'est aussi ce qui permet à ces étants [contenus dans le monde] d'être rencontrés spatialement (Heidegger, 1965, p. 137).

Ainsi considéré, le film, parce qu'il se détourne ou s'éloigne du sens du monde, retourne au sens du monde et de la pensée et impose, constamment, un questionnement éthique. Plus encore, le film, en retenant, en appelant et en dispersant le sens de la mondanéité et de la pensée, comprend le sens du mouvement et de la déchirure d'une pensée prise et aux prises non seulement avec le monde et sa compréhension, mais avec le moule de l'écriture filmique et des genres qui en découlent.

Mais on aimerait, avant de clore cette partie de l'analyse, revenir à quelques définitions du terme de Dasein en usage dans les écrits de Heidegger. "Cet étant que nous sommes chaque fois nous-mêmes et qui a, entre autres possibilités d'être, celle de questionner, nous lui faisons place dans notre terminologie sous le nom de Dasein. Le Da-sein [est ici considéré] comme étant tenu et entrant dans le rien qu'est l'être, entretenu comme rapport” (Heidegger, 1965, p. 31).

Il appartient essentiellement à l'être-là d'être au monde. La compréhension de l'être propre à l'être-là comprend donc tout aussi originellement la compréhension de ce qu'on nomme "le monde" et la compréhension de l'être de l'étant qui dans ce monde nous devient accessible (p. 29).

On le voit, le Dasein filmique peut être, de façon sauvage, tenu pour ce rapport, ce perpétuel battement transitif dans lequel se tient ou apparaît, hic et nunc, la pensée en écriture. Et elle peut être tenue, cette écriture, comme participant de l'apparition du monde et de la réalité que le Dasein traverse. "L'expression apparition peut elle-même signifier encore deux choses: d'abord l'ap-paraître au sens de s'annoncer et donc de ne-pas-se-montrer, elle signifie alors l'annonciateur lui-même; ce qui, sitôt qu'il se montre, dénonce quelque chose qui ne-semontre-pas. Et finalement on peut employer apparaître comme 
une dénomination du phénomène dans son vrai sens de semontrer" (Heidegger, 1986, p. 56).

En fait, il s'agit ici de concevoir l'expérience d'écrire l'image et le récit, et d'admettre simplement que le là correspond à l'expression d'une pensée dans l'appareil filmique. Le terme de $D a$ $\operatorname{sein}^{15}$ ne représente en définitive qu'une façon de mettre en évidence le cours de la pensée dans l'écriture, les termes de mondanéité et de momentanéité, une manière de chercher une association entre l'appareillage du film, spatio-temporel, et le monde que configure la pensée en écriture. On ne renvoie donc plus à cette vieille notion d'immanence des mondes dans le film ni même à l'impression de réalité, mais on soumet à l'analyse l'entreprise phénoménologique du film par laquelle ce qui est vu, c'est la manière de voir et d'appréhender les phénomènes. Malgré les détours qu'il emprunte, le cinéma nous convie à prendre part à une rhétorique du penser et de la faillibilité de son langage.

\section{Faillibilité du langage filmique}

Par exemple, si nous lisons dans notre livre du monde la description d'un meurtre, avec tous ses détails physiques et psychologiques, la pure description de ces faits ne contiendra rien que nous puissions appeler une proposition éthique. Le meurtre sera exactement au même niveau que n'importe quel autre événement, par exemple, la chute d'une pierre. Assurément, la lecture de cette description pourrait provoquer en nous la douleur, la colère ou toute autre émotion, ou nous pourrions lire quelle a été la douleur ou la colère que ce meurtre a suscitée chez les gens qui en ont eu connaissance, mais il y aura là seulement des faits, des faits, des faits mais non de l'éthique. Aussi, me faut-il dire que si je m'arrête à considérer ce que l'éthique devrait être réellement, à supposer qu'une telle science existe, le résultat me semble tout à fait évident. Il me semble évident que rien de ce que nous pourrions jamais penser ou dire ne pourrait être cette chose, l'éthique; que nous ne pouvons pas écrire un livre scientifique qui traiterait d'un sujet $[\ldots]$ d'un niveau supérieur à tous autres sujets. Je ne puis décrire mon sentiment à ce propos que 
par cette métaphore: si un homme pouvait écrire un livre sur l'éthique qui fût réellement un livre sur l'éthique, ce livre, comme une explosion, anéantirait tous les autres livres de ce monde (Wittgenstein, 1971, p. 146147).

Impossible donc d'écrire un livre qui soit vraiment sur l'éthique. Impossible, car celui-ci devrait porter sur la réalité ellemême, sur les langages de la réalité qui en fabriquent les interprétations et les dérives, sur la réalité en tant qu'elle a été modifiée par le langage et la pensée, sur l'utilité et l'idéalité du langage en tant qu'il peut être un indicateur de "vérité". Or, l'énoncé, à valeur de vérité absolue est, selon Wittgenstein, impossible puisque, notamment, il ne saurait être "transhistoriquement " indiscutablement vrai. Reste l'énoncé à valeur relative, l'énoncé qui, se pliant à maintes conditions vériconditionnelles, n'est que relativement vrai. Ces conditions comprendraient les rapports de l'énoncé avec le réel, l'imaginaire et la source langagière qui l'exprime. Et il devrait porter, ce livre sur l'éthique, sur l'idéation de la parole, sur le fait de ne pas pouvoir rendre compte par le langage de la charge indicible du langage et, enfin, sur l'expression de la pensée qui, parfois, ne se connaît pas elle-même. La démarche est, compte tenu de ce qui vient d'être dit, psychanalytique. Elle est aussi sociale, anthropologique, philosophique, historique, littéraire et cinématographique dans la mesure où l'une et l'autre de ces disciplines servent à révéler les indices et les incidences cachées des langages.

$\mathrm{Si}$, comme le soutient Wittgenstein, "Les limites de mon langage signifient les limites de mon [propre] monde" (1976, p. 141) et si "je suis mon propre monde" (p. 142), il faut croire que le monde et la pensée sont, comme l'éthique, logiquement restreints par le langage, même cinématographique. Ce qui, plus loin, faisait dire à Wittgenstein qu'il fallait "donner du front aux bornes du langage [...]" (Waismann, p. 150), "[...] affronter [au terme du propos éthique], les bornes du langage" (1971, p. 55), repenser la pensée, et les réalités, dans le site du discours.

Plus encore, et je retrace ici quelques-unes des contradictions que laisse profiler la citation de Wittgenstein en rapport avec le questionnement éthique du cinéma ici promulgué, le langage 
cinématographique ne saurait être, selon la règle de la prévoyance auparavant énoncée, purement descriptif. Même plutôt descriptifs, les syntagmes filmiques réferent à l'écriture et, par elle, à la manifestation, même occultée, d'un certain Dasein, d'une pensée. Or, ne pouvant pas être que purement descriptif, le langage cinématographique n'est évidemment pas pour autant éthique. Malgré le fait qu'il se moule, par son matériau, sur le monde, malgré le fait qu'il en joue l'immanence, le film n'est pas garant de la vérité du monde. Fictionnel, le film n'est pas hors le monde. Malgré, ici aussi, l'apparence d'ubiquité de son matériau qui fait traverser et traverse les lieux du monde et de la réalité, le film historique n'est pas plus à l'intérieur de l'histoire que les récits historiques écrits. Comme le soulignait Ricœur, qu'on cite plus loin, le travail - ici filmique - même le plus référentiel, le plus porté sur la réalité propose inévitablement la redescription de cette dernière. Il n'y a donc pas au cinéma de pure apparence.

On le voit, comme il en est des autres langages, le cinéma impose au regard de ses manifestations, de ses occultations et de ses simulacres un questionnement énonciatif, éthique. Pour poser les paramètres d'une éventuelle analytique du langage cinématographique et de son gabarit éthique, deux voies d'analyse pourraient être proposées. La première concerne le degré d'adhérence ou de charge référentielle du récit, qu'il soit fictionnel ou documentaire. La deuxième se préoccupe de l'inhérence et de la manifestation de la pensée dans l'écriture.

La première hypothèse comporte à son tour une double perspective. La première consiste à se demander, le plus simplement possible, comment se dessine au cinéma l'horizon référentiel de la fiction et du documentaire. La deuxième, complémentaire de la première, vise à vérifier la constitution d'un horizon référentiel qui, fictionnel, enjoint la réalité de le traverser et, à l'inverse, celle d'un horizon référentiel qui, documentaire, ne peut éviter la fictionnalisation de son discours. "[...] c'est alors, disait Ricœur, que pourra être montré de quelles manières différentes ma prétention référentielle du récit de fiction et celle du récit historique sont appropriées à la structure ontologique de l'historicité [et, c'est notre propos, de la mondanéité]. En d'autres mots, le [...] 
problème est de comprendre comment notre historicité [comme notre mondanéité] fondamentale est portée au langage par la convergence des différents modes de discours narratif, compte tenu de leurs prétentions référentielles différentes quoique complémentaires" (1980, p. 50).

Bien que ne soit pas ici mise au jeu la question de l'historicité et du cinéma qu'un propos éthique aurait, il est vrai, pu convoquer, cette perception de l'historicité et du récit rejoint celle de la mondanéité. Est, de fait, dans la mire de l'analyse celle d'une mondanéité que traversent, si l'on veut par suite, le sentiment de l'historicité et le récit historique. La mondanéité, elle, retient notamment la quotidienneté et, au cinéma, la réalité. Cette mondanéité filmique concerne, en somme, l'adhérence référentielle, celle de la réalité dans la fiction et celle de la fiction dans la réalité, qu'elle soit quotidienne ou historique, et il inclut, ce terme de mondanéité, l'inhérence de la pensée dans le monde de l'écriture qui configure ou mime ce dernier.

La mimésis, telle qu'envisagée entre autres par Ricœur, si elle constitue une métaphore de la réalité, se pose par l'écriture d'un matériau de photographie comme une métaphore, mais en acte (1980, p. 55). En acte, puisque celle-ci répète directement le monde en en renouvelant, autrement, les apparences. Or, le pathos langagier peut précisément tenir, pour ce qui concerne certains films de fiction et certains documentaires, de cette façon de métaphoriser le monde et le discours par la pensée, en (ex)posant l'un et l'autre, le monde et la pensée, comme absents, comme des simulacres de l'absence (1980, p. 55). Le propos éthique tient de ces apparences, du non-apparaître de l'apparition, du simulacre, de l'«indoublé", d'un discours de l'immanence supposée.

Mais nous avons vu que la référence de la fiction à l'irréel n'est que la contrepartie négative de sa référence productive; ou, pour le dire en d'autres termes, la suppression d'une référence de premier ordre - que nous avons appelée par convention la "description" du monde - est la condition de possibilité d'une référence de second ordre que nous appelons ici la redescription du monde. Une œuvre littéraire, me semble- 
t-il, n'est pas une œuvre sans référence, mais une œuvre avec une référence dédoublée, c'est-à-dire une œuvre dont l'ultime référence a pour condition une suspension de la référence du langage conventionnel (1980, p. 56).

Mais abordons cette fois-ci directement la question du référent, de la réalité, pour vérifer l'impact éthique de sa chute dans certains propos cinématographiques. On pourrait prétendre, et on aurait pour une large part raison, que, contrairement à la littérature - pensons pour en mesurer encore plus l'impact à la pornographie - c'est le noir et blanc de l'écriture qui produit le référent, alors qu'au cinéma ce sont des êtres de chair qui, tels des petits bouts de papier, se plient aux volontés de l'écriture. Au cinéma, pour ce qui est notamment de ce genre de production, c'est en quelque sorte la réalité qui descend ${ }^{16}$ dans l'écriture qui, en s'altérant, reconstruit le référent. Ce n'est plus l'inverse, ce n'est plus comme en littérature, l'écriture qui monte, et fait surgir le référent, la réalité.

Posant, de cette façon, du côté de l'éthique, la problématique référentielle et celle, sous-jacente, des genres, on est enclin à se questionner sur le sens de la réalité qui, même dans le documentaire, s'altère parce que celle-ci est écrite plutôt que décrite et elle s'altère aussi par l'habitude que nous avons de voir écrire du simulacre, de la présence repoussée, de l'absence supposée, de la fiction.

Abordant la question d'un Dasein, d'une pensée inhérente à l'écriture filmique, considérant le réseau d'inter-férence de la réalité dans le film et, plus loin, des genres filmiques qui tendent à opposer le documentaire et la fiction, on peut, je crois, mieux mesurer les enjeux éthiques du langage cinématographique. Et ceux-ci tiennent d'une inquiétude. On peut s'inquiéter que des films pornographiques continuent d'être vendus dans les arrière-boutiques - et que pour arriver à les produire on use d'hommes, de femmes et d'enfants. On peut s'indigner que des Drôles de vidéos soient sur nos écrans de télévision - et que, pour ce faire, la population prépare des petites mises en scène qui tiennent trop souvent du sadisme. Est aussi préoccupant le fait que des nouvelles présentées dans les bulletins télévisés ne 
soient pas crues - ou, au contraire, si facilement crues qu'on les tienne, sur les plans historique et politique, pour exhaustives, vraies, indiscutables. À force de faire descendre la réalité dans les images télévisées et les films, se peut-il qu'elle s'en trouve altérée, rompue au jeu du simulacre? À force d'en jouer, sans en avoir l'air, se peut-il que ce soit le réel, le monde, qui s'ajuste sur l'art du langage de l'image ou, pour employer d'autres termes, que celui-ci change, par méconnaissance ou insouciance ${ }^{17}$, la configuration des réalités sans mesurer la violence qui peut découler de cette méconnaissance ou de cette insouciance? Si les langages filmique et télévisuel expriment la pensée, ne faut-il pas s'interroger sur l'impensée du discours et sur ses termes? Se peut-il, enfin, que, comme il en est des autres langages, à force de jouer de la réalité et des pensées, le cinéma parvienne à énoncer une réalité dérivée, autre, une réalité sans mémoire ou dont la mémoire ne serait que textuelle, filmique, indicible et où la violence serait, pour être ainsi faussement autorisée, volontairement indépassée?

Beau texte qui déplace les interrogations et qui permet de penser le rapport de la production littéraire et / ou picturale avec, non pas directement la réalité, mais avec l'univers des signes culturels, instance médiatrice où s'organisent et se croisent des micro-discours, des micro-représentations, qui fait que, quoi que nous fassions, que nous écrivions, de la fiction ou de l'écriture historienne, nous travaillons toujours sur du déjà-là sur du déjà-dit, sur du réel déjà sémiotisé, figé, cristallisé, sur le déjà-là de la représentation. Nous ne pouvons pas nous échapper de l'intertexte et de l'interdiscours dans lesquels nous sommes pris (Robin, p. 38).

L'examen visait, en somme et en conclusion, à fractionner, pour examiner ce qu'on a dit être l'immanence filmique. La première fraction, si on peut s'exprimer ainsi, voulait aider cet examen de la fabrique ou de l'inhérence de la pensée dans l'écriture; la deuxième, celui, éthique, du sens du monde et de la réalité contenu dans l'écriture qui en perpétue la mémoire et, le faisant, en fixe les dérives. Il y a, de fait, "la mémoire contre la pétrification ou la mémoire-pétrification, tel est, en dernière analyse, l'en- 
jeu réel" (Robin, p. 68) du propos éthique. L'ouvrage de la pensée dans le film est un faire, un faire-mémoire ou un faire-oubli. Et en tant que faire, déjà, sans qu'il soit encore des violences contenues dans les récits, il impose un constant questionnement éthique.

\section{NOTES}

1 Paul Ricœur a sous-titré son ouvrage Finitude et culpabilité. L'homme infaillible. Le terme de faillibilité fait, en ce qui concerne la présente étude, d'abord référence à la présence d'un être-là du discours filmique ou à celle d'un scripteur à l'écran, aux possibles, comme aux échappées du discours filmique et non pas, directement, au clavier indifférencié de la "violence" et du " mal ". Notons que le terme de scripteur: "désigne l'actant émetteur du texte, l'écrivain écrivant qui se définit ou est défini par ce rôle" (Dictionnaire de la communication, p. 450). L'on peut rappeler également, et notamment, les deux paramètres définis par Ricardou dans ses Textiques qui, pour aller très vite, concernent la scripture et la texture. Certaines des réflexions de MarieClaire Ropars auraient pu aussi être rappelées. Une précision supplémentaire s'impose: l'intérêt d'user de ce terme vient, non pas de ce qu'il permet une quelconque ouverture à une théorie de la communication, mais de ce qu'il autorise la reconnaissance d'un écrivain écrivant, d'un écrivain en train d'écrire ou, pour être plus clair encore, d'une pensée en travail dans le discours fimique. Cela dit encore, j'ai maintes fois fait référence à cette notion et $\mathrm{y}$ ai vu une sorte de bretelle d'accès de l'écriture à l'étude de la pensée en écriture que l'enquête ici menée sur l'être-là du discours filmique précise. Dans, notamment, un récent texte intitulé "Le cinéma ou la nostalgie des mondes", j'examinais cette idée de la présence d'un scripteur à l'écran en la mettant en rapport avec le principe d'une mondanéisation, que j'énonçais. Je voulais par là orienter la lecture de l'écriture filmique vers l'émergence et, pour être plus précise, vers les conditions d'émergence des couches narratives du film et des mondes possibles. Étaient, dans une perspective phénoménologique, mises au jeu l'idée de la fabrique cognitive du film par le scripteur et celle, il va sans dire, de leurs entretiens respectifs avec le monde. ("L'expérience filmique. Approches philosophiques du cinéma " dans R.S.S.I., vol. 13, $\mathrm{n}^{\infty 1} 1-2,1993$, p. 243-256).

2 Cette pensée de Merleau-Ponty est reprise et expliquée par Jacques Dumont et Philippe Vandooren dans La Philosophie (Verviers: Les dictionnaires Marabout Université Savoir moderne), tome III, p. 539. Je cite plus avant: "Sa "dialectique" celle de Merleau-Ponty - concrète se fonde sur l'idée essentielle de l'ambiguïté de l'existence. Il montre la précarité, la contingence de l'émergence du sens à partir du non-sens (par exemple, du rationnel à partir de l'irrationnel, de la justice à partir de la violence...), la relation faite à la fois de tension et de complémentarité, qui unit sens et non-sens. L'aventure humaine, sous ces trois formes ou dans ses trois expressions les plus significatives (parole et communication, histoire, unité de la "nature" et de la "culture" dans le corps propre comme quasi-sujet signifiant), cette aventure n'est consacrée ni garantie par aucune téléologie divine, aucun ciel intelligible des essences ou des valeurs. Une "retombée" dans le non-sens, et la violence demeure notre possible indépassable. Rien ne nous a été promis, et "l'ordre humain" doit être conquis sur fond de désordre, qui nous rappelle le sentiment de notre contingence. » On aurait pu, dans cette perspective et à la suite des R. Girard et H. B. Vergote, ouvrir le questionnement éthique sur la violence comprise dans les grands courants politiques et moraux. "On pourrait, disait ce dernier, même envisager une histoire de la violence qui ne serait rien d'autre que l'histoire de sa méconnaissance et des formes de plus en 
plus méconnaissables et de plus en plus efficaces de cette même violence que cette même méconnaissance rend possibles" ("Esprit, violence et raison", Études, vol. 366, n 3, p. 363).

3 L'existentialité, en tant qu'elle constitue le champ d'étude de l'existence, s'occupe de l'ontologie, de l'explication des structures ontologiques qui concernent les causes, les lois de l'être en tant qu'être et de l'être au monde. Notons par ailleurs, et pour ponctuer davantage encore la différence entre une éthique métaphysique et une éthique disons langagière, que l'actuel projet s'écarte de la conception humaniste, puis éthique de Heidegger. "Tout humanisme se fonde sur une métaphysique ou s'en fait lui-même le fondement. [...] si l'on considère la manière dont est déterminée l'essence de l'homme, le propre de la métaphysique se révèle en ce qu'elle est "humaniste". De la même façon, tout humanisme reste métaphysique" ("Lettre sur l'humanisme", Questions III, Paris: Gallimard, 1946, p. 87). L'éthique ici envisagée a un caractère langagier. Et le langage fait, en quelque sorte, passer la pensée.

4 Je reprends ici la définition de Jean-Paul Resweber dans son ouvrage Le Questionnement éthique (Paris: Cariscript, 1990) p. 28.

5 Les termes privilégiés par Paul Ricœur sont autrement employés dans la mesure où la faillibilité, comme le pathos, concerne le discours et non pas seulement son contenu mythique et symbolique.

6 Houziaux disait, dans Le Désir, l'arbitraire et le consentement. Pour une éthique du tragique (Paris: Aubier Montaigne, 1973) : "Il convient [...] de s'interroger sur le mot arbitraire. On peut le ressentir comme une bienheureuse délivrance d'évaluation humaniste des normes et des choix. Arbitraire serait alors signe hautain et discret d'un autre qui nous parle, et dont nous observons avec confiance les indices et les indications, sans réclamer abusivement d'en démêler et d'en juger les raisons" (p. 11-12).

7 L'ouvrage Le Langage silencieux d'Edouard T. Hall est à ce propos assez révélateur.

8 Le terme fait référence, souvent de loin, à la théorie de Martin Heidegger. Il souligne que: "Traduire simplement, Das Da-sein par : l'être-là, c'est risquer d'interpréter cet existential dans le sens de la facticité sartrienne. L'homme n'est pas cet étant qui est le "là", c'est-à-dire jeté dans la contingence d'une existence donnée. Il est le "là" de l'être, celui qui permet à l'être d'être là, de se dévoiler hic et nunc" ("Lettre sur l'humanisme", Questions III, Paris: Gallimard, 1946, p. 96).

9 "Le mémorial-pensé-dans-l'être se différencie essentiellement d'une pure remémoration de l'histoire prise au sens de passé écoulé" ("Lettre sur l'humanisme", Questions III, Paris, Gallimard, 1946, p. 109).

10 Ce terme est en usage dans les travaux de Martin Heidegger. Selon l'auteur que je cite: "La mondanéité est un concept ontologique. Il désigne la structure d'un moment constitutif de l'être-au-monde. En nous interrogeant ontologiquement sur le "monde", nous ne quittons donc nullement le champ thématique proposé à l'analytique de l'être" (L'Étre et le temps, Paris: Gallimard, 1965, p. 87). Bref, la mondanéité fait écho à ce concept ontico (de l'être existant)-ontologique (de la compréhension de l'être en tant qu'être) ou à celui de l'être-là dans le monde. Le là de l'être-là pose la question des horizons de l'être et du lien entre le monde et l'être lui-même. On le voit, si le présent travail peut accueillir certaines prétentions phénoménologiques, on ne saurait par avance lui prêter, pour des raisons d'ordre épistémologique évidentes, des prétentions ontologiques.

11 On reprend là, pour jouer de ce concept, le titre d'un ouvrage de cet auteur.

12 L'adjoint du suffixe éité marque en quelque sorte la présence réconciliée de l'être dans le monde (mondanéité), le temps (temporellité) et l'histoire (historialité).Voulant aborder la question d'une éthique cinématographique, langagière, on évite 
d'aborder le débat entourant les ambiguittés politiques de l'auteur. Sans questionner davantage ses propos, notons que, selon Janicaud, quatre périodes se dessinent chez Heidegger. Une première période a-politique (c'est celle correspondant à la publication de l'ouvrage L'Etre et le temps dont on fait presque uniquement usage). Une seconde a un fondement politique ontologique. Une troisième marque une transition, ambiguë, vers une sorte de retour sur une a-politique historiale - quatrième période (Éliane Escoubas reprend les propos de Janicaud dans "L'ombre de cette pensée. Heidegger et la question politique», Les Temps modernes, $\mathrm{n}^{*}$ 536-537, mars-avril 1991, p. 61-69).

13 Voir à ce propos La Chambre claire de Roland Barthes. Je n'approfondis pas davantage ce rapport entre le Dasein et l'espace photographique. Je préfere réserver, pour un second article, l'examen des horizons de temps dans l'espace photographique du film.

14 Ces termes sont de Heidegger. On en fait un usage différent en s'inspirant des concepts qu'ils comprennent.

15 Il est dit, dans L'Être et le temps (Paris: Gallimard, 1965): "Si l'être-là ne peut faire retour sur lui-même qu'à partir de son être-auprès-de [...] l'étant intramondain, son "ici" ne pourra, lui aussi, se découvrir qu’à partir du "là " [...] contenu dans le terme d'“ être-là " correspondant à un da (Dasein), et non à un dort (là-bas) dont il est question ici par opposition à un hier (ici)" (p. 297). Notons que René Lindekens a fait référence à la présence du Dasein dans l'image. Cette expérience de l'image s'inscrit, en ce qui le concerne, dans un chapitre intitulé "Image mentale et code de verbalisation ". On le voit, l'idée de la description d'une pure expérience ne concerne pas la représentation ni la manifestation de la pensée mise en image, mais les images mentales elles-mêmes (Dans l'espace de l'image, Paris: Aux amateurs du livre, 1986, p. 27). Le Dasein ici considéré en tant qu'il est là concerne la pensée en manifestation dans l'écriture filmique.

16 C'est à peu de choses près le propos des recherches filmiques de Paul Warren.

17 Je me permets de renvoyer le lecteur à la deuxième note infrapaginale.

\section{OUVRAGES CITÉS}

Dumont, Jacques et Vandooren, Philippe. La Philosophie. Bruxelles: Les Dictionnaires Marabout Université Savoir moderne, tome III, 1972.

Escoubas, Éliane. "L'ombre de cette pensée. Heidegger et la question politique ". Les Temps modernes, $\mathrm{n}^{\text {oa }}$ 536-537 (mars-avril 1991) p. 61-69.

Heidegger, Martin. Acheminement vers la parole. Paris: Gallimard, 1984.

Heidegger, Martin. Etre et temps. Paris: Gallimard, 1986.

Heidegger, Martin. L'Être et le temps. Paris: Gallimard, 1965.

Heidegger, Martin. "Lettre sur l'humanisme», Questions III. Paris: Gallimard, 1946, p. 73-154.

Houziaux, Alain. Le Désir, l'arbitraire et le consentement. Pour une éthique du tragique. Paris: Aubier Montaigne, 1973.

Lindekens, René. Dans l'espace de l'image. Paris: Aux amateurs du livre, 1986.

Resweber, Jean-Paul. Le Questionnement éthique. Paris: Cariscript, 1990.

Ricœur, Paul. «La fonction narrative ", La Narrativité. Paris: Centre national de la recherche scientifique, 1980, p. 49-68.

Ricœur, Paul. Finitude et culpabilite. L'homme faillible. Paris: Montaigne, 1960.

Robin, Régine. Le Roman mémoriel. Québec: Préambule, 1989. 
Roy, Lucie, "La nostalgie ou l'écriture des mondes du cinéma ", "L'expérience filmique. Approches philosophiques du cinéma ", R.S.S.I., Vol. 13, n 1-2 (1993), p. 243-256.

Truxillon, Jean-Paul et Corsa, Philippe. Dictionnaire de la communication. Paris: Armand Colin, 1991.

Vergote, Henri-Bernard. "Esprit, violence et raison", Etudes, vol. 366, n 3 (1987) p. 363-374.

Waismann, Friedrich. "Les vues de Wittgenstein sur l'éthique", Leģons et conversations. Paris: Gallimard, 1971, p. 155-159.

Wittgenstein, Ludwig. "Conférence sur l'éthique ", Leçons et conversations. Paris: Gallimard, 1971, p. 141-159.

Wittgenstein, Ludwig. Tractatus logico-philosophicus. Paris: Gallimard, 1976. 